\title{
Das Gesundheitswesen darf viel, könnte aber auch weniger kosten
}

\section{Max Geiser}

1 Günter P. Warum versagt die Ärztelobby? Schweiz Ärztezeitung 2004;85(15):765-66.

2 Sommer JH. Muddling Through Elegantly: Rationierung im Gesundheitswesen. Basel: EMH Schweizerischer Ärzteverlag; 2001

3 Angaben des Eidg. Statistischen Amtes über die Zusammensetzung des Bruttoinlandproduktes.

12 Kocher G. Zweitteuerstes Gesundheitswesen der Welt. Schweiz Ärztezeitung 2004;85:2142-3.

* Für das Jahr 2002 betrugen die Ausgaben für das Gesundheitswesen in der Schweiz 48 Milliarden Franken, was 11,2\% des Bruttoinlandproduktes ausmacht. Die Kosten für die Grundversicherung werden vom Bundesamt für Gesundheit für das Jahr 2003 mit über 18 Milliarden Franken angegeben (NZZ vom 23./24.7.2004) Im Vergleich mit anderen Industrieländern hat die Schweiz das zweitteuerste Gesundheitswesen hinter den USA [12].

Korrespondenz:

Prof. Dr. med. Max Geiser

Gossetstrasse 49

CH-3084 Wabern
Die heute in zivilisierten Industrieländern lebenden Menschen haben eine grössere Lebenserwartung, müssen weniger arbeiten, geniessen mehr Freizeit und Beschäftigungsmöglichkeiten und eine bessere Gesundheit als die Menschen aller vorausgegangenen Generationen. Kein anderes Gebiet menschlicher Tätigkeit hat während der letzten 100 Jahre mehr zur Verbesserung der menschlichen Lebenssituation beigetragen als die sich vom dogmatischen Denken zunehmend befreiende, naturwissenschaftlich fundierte Medizin. Diese stützt sich auf die Ergebnisse der Erforschung der Natur innerhalb und ausserhalb des Menschen und sucht nach den Ursachen von Krankheiten - auch derjenigen des Gehirns. Sie erstrebt die Heilung und Verhütung von Krankheiten und Verletzungen und wenn möglich die Elimination von Seuchen und Krankheiten. Heute wird leicht vergessen, dass dank der Erforschung der Krankheitsursachen in der kurzen Zeit von lediglich ungefähr 150 Jahren viele Seuchen, Tetanus, Diphtherie, Tollwut und Poliomyelitis praktisch verschwanden und Tuberkulose und Lepra, Malaria, Osteomyelitis, Skorbut, Rachitis, Struma und Karies in die Schranken gewiesen werden konnten. Die Folgen von noch unheilbaren Krankheiten und nicht verhüteten Verletzungen und das Sterben wurden erträglicher gemacht.

Heute gehört die Befriedigung der menschlichen Grundbedürfnisse im Bereiche der Gesundheit zu den Aufgaben eines vernünftig funktionierenden Staates. Zu den Grundbedürfnissen gehören die Verhütung von Seuchen mit Hilfe der Versorgung mit einwandfreiem Wasser, der Kanalisation, der Nahrungsmittelkontrolle und Impfungen, die medizinischen Leistungen in Notfällen und die Behandlung von schweren Krankheits- und Verletzungsfolgen. Es darf jedoch nicht übersehen werden, dass die Inanspruchnahme von medizinischen Leistungen nicht vor allem zur Befriedigung von Grundbedürfnissen wegen bestehenden Krankheitsbereitschaften und Lebensumständen erfolgt, sondern zunehmend zur Befriedigung von individuell unterschiedlichen Ansprüchen im Rahmen der persönlichen Vorstellungen über Gesundheit und Wohlbefinden.

\section{Die Vielfalt der Kostenverursacher - Grenzenlosigkeit des Wünschbaren}

In der Diskussion über die Ursachen der steigenden Kosten des Gesundheitswesens betonen Politiker und viele Ärzte die Rolle des «medizinischen Fortschrittes» [1]. Ökonomen [2] und Politiker betrachten die Ärzte als kostentreibende Anbieter. Alle drei übersehen die Auswirkungen der Ausweitung des Gesundheitsbegriffes über die Abwesenheit von ernsthaften Krankheiten hinaus auf das «vollkommene physische, psychische und soziale Wohlbefinden». Diese Ausweitung hat dazu geführt, dass es keine Grenzen mehr für das Wünschbare in Diagnose, Therapie, Vorsorge, Komfort und Wohlbefinden gibt, wenn es um die Erhaltung und Wiederherstellung der Gesundheit geht. Ferner übersehen sie die ungenügende Überprüfung des solidarisch finanzierten Leistungskatalogs der Grundversicherung auf Wirksamkeit und Notwendigkeit und den zu kleinen Anteil der kostenbegrenzenden direkten Finanzierung von unwirksamen und überflüssigen Gesundheitsmarktangeboten durch den Konsumenten. All dies führt dazu, dass jedes noch so grosse Angebot ausgeschöpft wird. Ausserdem wird gerne vergessen, dass ein liberaler Lebensstil in einem langen Leben nicht nur mit Freuden, sondern auch mit Gesundheitsrisiken verbunden ist und dass die Alterskrankheiten noch nicht heilbar und eliminiert sind. Die Bemühungen zur Milderung der Folgen von eingegangenen Gesundheitsrisiken und der noch nicht verstandenen und nicht heilbaren Alterskrankheiten kosten viel.

Für eine sachliche Beurteilung der Kosten des Gesundheitswesens muss zur Kenntnis genommen werden, dass der finanzielle Aufwand für begehrte Aktivitäten im Rahmen eines liberalen Lebensstils mit viel Mobilität, Spiel und Sport, Kommunikation und Medien, Mode, Ess-, Trink-, Tabak-, Drogen- und Sexlustbarkeiten, Wohnung und Bekleidung etwa siebenmal grösser ist als der finanzielle Aufwand für das Gesundheitswesen, wenn die Angaben des Eidgenössischen Statistischen Amtes [3] über die prozentuale Zusammensetzung des Bruttoinlandproduktes korrekt sind.* Die Kosten für die genannten begehrten Aktivitäten werden vom Konsumenten entspre- 
chend seinen unterschiedlichen Neigungen und finanziellen Möglichkeiten im freien Markt direkt finanziert. Angesichts dieser Tatsache wäre eigentlich zu erwarten, dass der vernünftige Bürger die viel geringeren und zu einem guten Teil selbst verursachten Kosten für reparierende Gesundheitsmassnahmen für tragbar beurteilen würde und bereit wäre, Unwirksames und Überflüssiges aus der solidarischen Finanzierung herauszunehmen und dem freien Markt zu überlassen. Die Diagnose des Gesundheitsökonomen [2], wonach die Situation im Gesundheitswesen so gravierend sei, dass sich die Frage nicht stelle, ob, sondern wie die Ausgaben für das Gesundheitswesen rationiert werden sollen, um das Problem der knappen Mittel in den Griff zu bekommen, ist voreilig. Der Ökonom übersieht, dass es sich beim Gesundheitsbereich um die wichtigste Sphäre des Individuums handelt und dass eine Rationalisierung des Leistungskatalogs und der Finanzierung der Leistungen bis heute nicht realisiert wurde.

\section{Unterschiedlicher Umgang mit den Kosten des Gesundheitswesens}

Es gibt drei Möglichkeiten:

\section{Den Gesundheitsmarkt unverändert blühen lassen}

Wenn man den blühenden Gesundheitsmarkt samt seinen Auswüchsen [4] für erfreulich hält [1], geht es darum, das Gesundheitswesen vor anderen solidarisch finanzierten Ausgaben zu privilegieren. Die Sicherstellung der Finanzierung mit Hilfe der Erhöhung der Mehrwertsteuer [1] ist ein ökonomisch nicht akzeptabler Vorschlag.

\section{2. Überprüfung des solidarisch finanzierten Leistungskatalogs der Krankenversicherung} Wenn im Interesse der Patienten, der Glaubwürdigkeit der Heilkunde und der Akteure im Gesundheitswesen und im Interesse der Ökonomie nur Leistungen mit erwiesener Wirksamkeit solidarisch finanziert werden dürfen (Art. 32 KVG), drängt sich die Überprüfung des Leistungskataloges auf Wirksamkeit und Notwendigkeit auf. Dabei muss zwischen der Forderung nach «Qualitätssicherung» und der Prüfung nach Wirksamkeit unterschieden werden. Die Qualitätssicherung gilt in der wissenschaftlich fundierten Medizin als selbstverständliche Aufgabe, um den Patienten vor Schäden zu bewahren. Sie muss jedoch von der Prüfung der Wirksamkeit medizinischer Massnahmen unterschieden werden.

Denn auf Irrtum oder Aberglaube beruhende unwirksame Praktiken verursachen hohe Kosten, auch wenn sie nach den Vorschriften der Erfinder «qualitätsgesichert» durchgeführt werden.
Im Bereich der Therapie der Krankheits- und Verletzungsfolgen ist die Durchführung von möglichst objektiven Wirksamkeitsprüfungen von alten und vor allem von neuen Praktiken, abgesehen von den meisten Medikamenten, noch nicht die Regel [5, 6]. Zum Beispiel hat bis heute noch niemand untersucht, ob nach einem Gelenkersatz immer ein Rehabilitationsaufenthalt für das Behandlungsergebnis wichtig, unbedeutend oder sogar schädlich ist oder welche Vorsorgeuntersuchungen wirklich sinnvoll sind, Leerlauf bedeuten oder sogar Angst und unnötige Kosten verursachen und wie regelmässige Check-ups zu einer kostenträchtigen Gesundheitsbesessenheit führen. Erwiesene wirkungslose, fragwürdige und irreführende Praktiken sollten wie andere vom Konsumenten direkt finanzierte Güter dem freien Markt überlassen werden. Dies benachteiligt diejenigen Bürgerinnen und Bürger nicht, die sich solche Praktiken nicht leisten können oder wollen. Sie entgehen vielmehr medizinischen Torheiten und Modeerscheinungen und deren Nebenwirkungen. Leichtgläubige, von Gesundheitsmagazinen desinformierte oder mystisch veranlagte Bürger haben dagegen die Möglichkeit, ihre Gesundheitsvorstellungen bei Placebospezialisten selbstfinanziert zu befriedigen.

\section{Unterstützung der Krankheits- ursachenforschung}

Die Verbesserung der Behandlung der Folgen von noch nicht heilbaren Krankheiten und von nicht verhüteten Verletzungen ist zweifellos erwünscht. Dieser «medizinische Fortschritt» kostet jedoch viel und löst trotz «Technologieexplosion» die Probleme selbst mit «Spitzenmedizin» in Form von Organ-, Gefäss- und Gelenkersatz nicht vollständig. Diese Reparationsmedizin wird deswegen korrekterweise als «half-way technology» bezeichnet. Die Geschichte der Medizin lehrt [7], dass der grösste Beitrag zur Gesundheit aller Menschen unabhängig vom Alter, von der Begabung und vom sozialen Status und die grössten Kosteneinsparungen im Gesundheitswesen durch die Elimination von Seuchen und Krankheiten und durch die Verminderung von Verletzungen erreicht werden können. Die erst seit etwa 150 Jahren systematisch betriebene biomedizinische Erforschung der Krankheitsursachen befolgt diesen Weg mit bereits bemerkenswerten eingangs erwähnten Ergebnissen. Es trifft nicht zu, dass eine eliminierte Krankheit durch eine andere ersetzt wird und sich die wissenschaftlich fundierte Medizin ihren Grenzen nähere [8]. Sie steht nicht am Ende, sondern wahrscheinlich erst am Anfang des Verständnisses der Natur im
8 Zeyer A. Wenn Heilung nicht mehr möglich ist. NZZ, 25.8.2004. 
Molekularbereich. Nicht die reparierende Medizin, sondern die Medizin, die Krankheiten eliminiert oder dauernd heilt, verdient den Namen Spitzenmedizin. Die Erhöhung der öffentlichen und privaten Investitionen in die Grundlagenforschung wurde bereits vor 30 Jahren zur Lösung des Kostenproblems im Gesundheitswesen empfohlen [7]. Sollte idealerweise aus dem Homo sapiens schliesslich eines fernen Tages eine «disease free species» [9] werden, könnten sich die Ärzte darauf beschränken, alle diejenigen anspruchsvollen Mitbürger zu umsorgen, die, obwohl frei von ernsthaften Krankheiten, sich immer noch nicht wohl genug fühlen.

\section{Schlussfolgerungen}

Die Klagen über die Kosten des Gesundheitswesens, des erfolgreichsten Bereiches menschlicher Aktivität, und der Widerstand, den Katalog solidarisch finanzierter medizinischer Leistungen rigoros auf Wirksamkeit und Notwendigkeit zu überprüfen, um Unwirksames, Fragwürdiges und Bizarres der direkten Selbstbezahlung durch den Konsumenten im freien Markt zu überlassen, signalisieren, dass in der Gesundheitspolitik dogmatisches, irrationales Denken immer noch über das vernünftige, kausal verstehende Denken dominiert. Da es sich beim Gesundheitsbereich um die wichtigste Privatsphäre des Individuums handelt, ist es höchste Zeit, die in weniger wichtigen Lebensbereichen geforderte Selbstverwirklichung auch auf den Bereich der persönlichen Gesundheit auszudehnen. Denn es ist nicht einzusehen, weshalb der liberale Bürger, der einen risikoreichen Lebensstil frei wählen darf und selbst finanziert, nicht auch imstande sein sollte, zu entscheiden, mit wieviel Gesundheit er über die Befriedigung der Grundbedürfnisse hinaus zufrieden ist und wieviel ihm eine noch mögliche Steigerung seines Wohlbefindens verglichen mit anderen selbstfinanzierten wünschenswerten Dingen wert ist. Und er sollte sich nicht über die Kosten von medizinischen Massnahmen beklagen, die für die Verbesserung der Lebenssituation aller Bürger besonders wichtig sind und die sich um die Reparatur der gesundheitsschädigenden Nebenwirkungen des spassigen, risikoreichen Lebensstils bemühen.

Die ärztliche Behandlung entspricht nicht dem Verkauf eines standardisierten Industrieproduktes. Da das Behandlungsergebnis besonders bei technisch anspruchsvollen Massnahmen von der Kompetenz des Heilkundigen und den Eigenheiten des Patienten entscheidend beeinflusst wird, gehört es zur Selbstverwirklichung des freien, selbstverantwortlichen Bürgers, dass er den Arzt seines Vertrauens frei wählen darf. Wenn der Arzt jedoch immer mehr in die Doppelrolle als Beauftragter des Staates oder der Versicherung und als Partner des Patienten hineingezwängt wird, um so schwieriger wird es für ihn, sich masslosen Wünschen des Patienten zu widersetzen, selbst wenn er damit ausschliesslich das Interesse des Patienten im Auge hat. Der Patient ist seinerseits nicht daran interessiert, gegen übertriebene, risikoreiche oder leerlaufende Massnahmen zu opponieren, weil diese von der Versicherung bezahlt werden [10]. Dies führt zur Störung der natürlichen Partnerschaft zwischen dem Patienten und seinem Arzt und zum Verlust der vertrauensvollen Loyalität Im Arzt-Patienten-Verhältnis. ** Ausserdem verursacht dies steigende Kosten.

Wunschdenken und Gesundheitsbesessenheit, die durch die Ausweitung des Gesundheitsbegriffes über die Abwesenheit von ernsthaften Krankheiten hinaus in immer weitere Kreise der Bevölkerung dringt, erschweren Bemühungen, ein Gesundheitswesen massvoll und trotzdem wirkungsvoll zu gestalten. Gegenüber neuen Praktiken, die ungenügend auf Wirksamkeit geprüft auf den Markt drängen, ist Skepsis und Zurückhaltung notwendig.

Angesichts der zunehmend aufwendigeren Möglichkeiten der Reparaturmedizin ist das Ausbildungsniveau der Heilkundigen noch nie so viel wichtiger gewesen als ihre Zahl. Aber seit 40 Jahren sehen Bildungs- und Gesundheitspolitiker nie genug Maturanden und Ärzte. Trotz wiederholten Warnungen vor dem Abfall des Niveaus in Gymnasien und Universitäten blieben Politiker offenbar der Meinung, viele billige Heilkundige seien gut genug für obrigkeitsgläubige Bürger. Innerhalb und ausserhalb des Spitals sind gut ausgebildete, unabhängige Heilkundige notwendig, die das grosse Selbstheilungs- und Regenerationspotential des menschlichen Organismus respektieren, unnötige Behandlungen vermeiden, Patienten als einmalige Individuen mit gesundem Menschenverstand und Weisheit und nicht schematisch als Fälle im servilen Leistungserbringerstil behandeln. Sie sorgen auch dafür, dass eine Behandlung nicht schwerer zu ertragen ist als die Erduldung der Krankheit und dass das natürliche Sterben nicht quälerisch verzögert wird.

Die Förderung der Forschung mit dem Ziel, Krankheiten zu heilen und zu eliminieren und Verletzungen zu verhüten, dient der Gesundheit aller Menschen unabhängig von Alter, Begabung und sozialem Status und führt zur grössten Kosteneinsparung im Gesundheitswesen. lichkeit sei, die den Patienten nie im Stich lässt und auf gegenseitigem Vertrauen aufbauen kann. 